\title{
DMSO-Induced Denaturation of Hen Egg White Lysozyme
}

\author{
Ilja K. Voets, ${ }^{*} \dagger$ Willemberg A. Cruz, ${ }^{\ddagger}$ Christian Moitzi, ${ }^{\dagger}$ Peter Lindner, \\ Elizabeth P. G. Arêas, ${ }^{\ddagger}$ and Peter Schurtenberger ${ }^{\dagger, \|}$ \\ Adolphe Merkle Institute, University of Fribourg, Route de l'Ancienne Papeterie, P.O. Box 209, \\ CH-1723 Marly 1, Switzerland, Departamento de Química Fundamental, Instituto de Química, Universidade de \\ São Paulo, Av. Prof. Lineu Prestes 748, CEP 05508-000 São Paulo, SP, Brazil, and Institut Max von \\ Laue-Paul Langevin, F-38042 Grenoble Cedex 9, France
}

\begin{abstract}
We report on the size, shape, structure, and interactions of lysozyme in the ternary system lysozyme/DMSO/ water at low protein concentrations. Three structural regimes have been identified, which we term the "folded" $\left(0<\varphi_{\text {DMSO }}<0.7\right)$, "unfolded" $\left(0.7 \leq \varphi_{\text {DMSO }}<0.9\right)$, and "partially collapsed" $\left(0.9 \leq \varphi_{\text {DMSO }}<1.0\right)$ regime. Lysozyme resides in a folded conformation with an average radius of gyration of $1.3 \pm 0.1 \mathrm{~nm}$ for $\varphi_{\text {DMSO }}<$ 0.7 and unfolds (average $R_{\mathrm{g}}$ of $2.4 \pm 0.1 \mathrm{~nm}$ ) above $\varphi_{\text {DMSO }}>0.7$. This drastic change in the protein's size coincides with a loss of the characteristic tertiary structure. It is preceded by a compaction of the local environment of the tryptophan residues and accompanied by a large increase in the protein's overall flexibility. In terms of secondary structure, there is a gradual loss of $\alpha$-helix and concomitant increase of $\beta$-sheet structural elements toward $\varphi_{\text {DMSO }}=0.7$, while an increase in $\varphi_{\text {DMSO }}$ at even higher DMSO volume fractions reduces the presence of both $\alpha$-helix and $\beta$-sheet secondary structural elements. Protein-protein interactions remain overall repulsive for all values of $\varphi_{\text {DMSO }}$. An attempt is made to relate these structural changes to the three most important physical mechanisms that underlie them: the DMSO/water microstructure is strongly dependent on the DMSO volume fraction, DMSO acts as a strong H-bond acceptor, and DMSO is a bad solvent for the protein backbone and a number of relatively polar side groups, but a good solvent for relatively apolar side groups, such as tryptophan.
\end{abstract}

\section{Introduction}

It is well-known that the addition of organic solvents to aqueous solutions of proteins greatly affects the conformation of these biomacromolecules. However, a detailed description of the physical mechanism that drives these conformational changes is generally absent. Here, we present an in-depth study on the changes in lysozyme size, shape, conformation, and protein-protein interactions in aqueous DMSO mixtures within the full range of mixing fractions: for DMSO volume fractions,

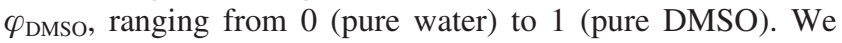
have chosen to focus on the organic solvent DMSO as we have observed a sol-gel transition in the ternary system lysozyme/ DMSO/water above a critical concentration of both lysozyme and DMSO. ${ }^{1,2}$ To better understand the physical mechanism behind this sol-gel transition, as well as the rheological properties and the microstructure of the final transparent gels, we need to understand what happens to the individual proteins upon an increase of the DMSO volume fraction. Besides, DMSO-induced structural transitions in proteins are of great practical interest as DMSO is used for example as cryoprotector ${ }^{3-5}$ and transporter of pharmaceuticals through the human skin and cell membrane. ${ }^{6,7}$

In this contribution, we will show that three distinct regimes of overall protein conformation and protein-protein interactions can be distinguished (Figure 1). In the first regime corresponding

\footnotetext{
* Corresponding author. E-mail: ilja.voets@unifr.ch.

University of Fribourg.

* Universidade de São Paulo.

Institut Max von Laue-Paul Langevin.

"Current address: Division of Physical Chemistry, Center for Chemistry and Chemical Engineering, Lund University, SE-221 00 Lund, Sweden.
}

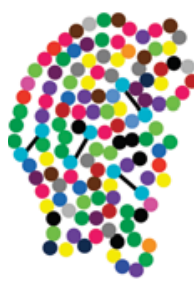

$$
\begin{gathered}
\text { native } \\
0 \leq \varphi_{\text {DMSO }}<0.7
\end{gathered}
$$

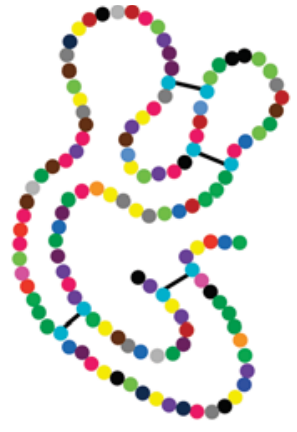

unfolded

$0.7 \leq \varphi_{\text {DMSO }}<0.9$

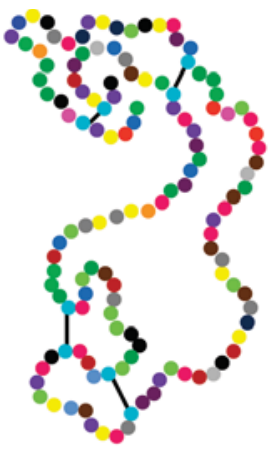

partially collapsed $0.9 \leq \varphi_{\text {DMSO }} \leq 1.0$
Figure 1. Artist impression of DMSO-induced denaturation in lysozyme/DMSO/water. Throughout this Article, these states will be referred to as "(quasi-)native", "unfolded", and "partially collapsed".

to $0<\varphi_{\text {DMSO }}<0.7$, the protein retains (to a certain extent) a (quasi-)native secondary and tertiary structure keeping the amino acids with hydrophobic residues deeply buried within the core of the macromolecule. We refer to the protein conformations in this regime as "folded". In the second regime, $0.7 \leq \varphi_{\text {DMSO }}$ $<0.9$, there is basically no difference in free energy between the folded and unfolded states, so that the structures are highly flexible and the hydrophobic residues become exposed to the solvent. We call these conformational states "unfolded" states, as the protein's characteristic tertiary structure is no longer observable. In the third regime, corresponding to $0.9 \leq \varphi_{\text {DMSO }}$ $<1.0$, the protein attempts to minimize the contact area between DMSO and many of its amino acids for which DMSO is a bad solvent: some of the residues that were buried in the core of 
the protein in regime I now become exposed, and vice versa, some of the residues that were previously exposed to the solvent may now become buried in the protein core. In this regime, we refer to the conformational state of the protein as "partially collapsed". The four disulfide bonds present in lysozyme connecting eight cysteins (in positions 6 and 127, 30 and 115, 64 and 80, and 76 and 94) greatly limit the extent of the DMSOinduced structural transitions. As the disulfide bonds serve to stabilize the native structure, ${ }^{8-10}$ they particularly hinder the realization of the "quasi-inverted" structures, that is, the partially collapsed states in regime III. Therefore, we do not observe the anticipated globular-random coil-globular shape transformation upon increasing $\varphi_{\text {DMSO }}$ in our small angle neutron scattering experiments. It is only the folding-unfolding transition that yields significant changes in overall shape and tertiary structure. By contrast, the protein-protein interactions, and to a lesser extent lysozyme size, are affected by both transitions. They exhibit a complex, nonmonotonous dependence on $\varphi_{\text {DMSO }}$, but remain overall repulsive for all $\varphi_{\text {DMSO }}$.

The physical mechanisms driving the structural changes described above can be dissected into three contributions that operate in a synergistic manner. First, the addition of DMSO to water has a profound effect on the solvent microstructure ${ }^{5,11}$ and on the number of water molecules H-bonded to each other ${ }^{12}$ resulting in a number of very interesting phenomena, the origin of which has been much debated. The formation of eutectics, ${ }^{13}$ the formation of (stable) $\mathrm{H}_{2} \mathrm{O}-$ DMSO hydrates, $5,12,14,15$ and the occurrence of significant DMSO self-association have all been suggested. ${ }^{11,14,16,17}$ The fact is that curves describing the dependence of several physical and spectroscopic parameters of that binary mixture as a function of the organic solvent concentration consistently show peculiar features such as discontinuity, curve inflections, or maxima/minima at $\varphi_{\text {DMSO }} \sim$ $0.63-0.75 .5$ Examples include characteristic Raman frequencies, NMR relaxation times, refractometric data, and conductometric data. ${ }^{1,11}$ Several other organic-aqueous systems in which the binary mixture can be represented by an aprotic polar solvent in water likewise exhibit such features. ${ }^{1,11}$ The microheterogeneous character of these binary mixtures has been acknowledged by several authors, and various techniques have been employed in their investigation, such as mass spectrometry and solvatochromic probing. ${ }^{16-18}$ Second, the sulfoxide groups of DMSO act as competitors of the polypeptide backbone and side chain carbonyl groups when it comes to intra- and intermolecular H-bond formation. Third, DMSO is a bad solvent for the backbone of the protein as well as for a number of relatively polar side groups, while it is a good solvent for relatively apolar side groups, such as those found in amino acids with aromatic rings, such as tryptophan. ${ }^{19}$

Hence, we propose the following model for DMSO-induced denaturation of lysozyme. In regime I, $\varphi_{\text {DMSO }}<0.7$, an increase in $\varphi_{\text {DMSO }}$ increasingly disrupts (predominantly) intramolecular hydrogen bonds between carbonyl and amino groups in the protein backbone (secondary structure) and side groups (tertiary structure). Still, overall there is a pronounced energetic penalty against exposure of hydrophobic units to the solvent mixture, and the protein retains a semiglobular shape with its hydrophobic units buried. In the second regime, $0.7 \leq \varphi_{\text {DMSO }}<0.9$, this energetic penalty is fully absent. Differential scanning calorimetry experiments no longer register a melting transition: the unfolding enthalpy vanishes around $\varphi_{\text {DMSO }} \sim 0.75$, while the melting temperature approaches room temperature. ${ }^{20-23}$ As a large number of intramolecular hydrogen bonds have already been broken, the protein adopts a very flexible, floppy structure where both polar and apolar residues are exposed to the solvent. In regime III, $\varphi_{\text {DMSO }} \geq 0.9$, the amount of DMSO present in the system is so large that the protein will now attempt to minimize the contact area between DMSO and residues for which DMSO is a bad solvent, so that it will rearrange in such a way as to expose the units that were buried in regime I and bury those that were exposed to the solvent in regime I. In contrast to the first regime, due to the presence of a large amount of the strong H-bond acceptor DMSO, H-bonding between carbonyl and amino groups in the protein backbone is only marginally involved in this "inverse folding" process. Instead, it is the minimization of surface tension that is responsible for the transition between the unfolded state in regime II and the newly formed conformation which we term "partially collapsed", in analogy to the term employed for synthetic polymers in bad solvent conditions (see for example refs 24-26). Indeed, we might even speculate that, as the H-bonding in regime I promotes burying of apolar residues in the inner protein cavity, the absence of $\mathrm{H}$-bonds between these same units is a necessary prerequisite to be able to keep them exposed to the solvent. Note that the number of possible unfolded and partially collapsed conformations is still restricted by the four intact disulfide bridges within lysozyme. In summary, the folding (regime I) - unfolding (regime II) transition and the unfolding (regime II) - partially collapsed (regime III) transition are essentially driven by the propensity of DMSO to alter the structural arrangement of and $\mathrm{H}$-bonding between water molecules on the one hand (indirect effects) and to compete with the amino acids for H-bonds and to solvate apolar side chains on the other hand (direct effects). DMSO thus affects both the conformational and solution stability of lysozyme.

While the effect of DMSO on the secondary and tertiary structure of lysozyme has been well documented,,$^{1,27-30}$ little is known about DMSO-induced changes in lysozyme size, shape, and interactions. In the following, we will provide the reader with a detailed description and in-depth discussion of the experimental results obtained in this study as well as previously reported results of several other groups taken from the literature. It is from the careful consideration of all of these experimental observations that a consistent physical interpretation of DMSOinduced denaturation of lysozyme has finally emerged. Although the universality of the results for other proteins and organic solvents remains to be proven, we would like to note that several other ternary systems display similar features (see for examples refs 31-34). We hope that our understanding of this particular system can serve as a basis for a detailed understanding of conformational changes in a great number of other protein/ organic solvent/water systems. Finally, we would like to note that the addition of an organic solvent to a protein solution can be an efficient way of shifting the balance between various conformational states: a necessary first step on the way toward answering the important question whether certain conformational states are more prone to aggregation than others.

\section{Experimental Section}

2.1. Materials. Hen egg white lysozyme (L6876, LOT $117 \mathrm{~K} 1547)$ and DMSO were purchased from Sigma and used without further purification. Deuterated solvents, $\mathrm{D}_{2} \mathrm{O}$ and $d_{6^{-}}$ DMSO were purchased from Cambridge Isotope Laboratories and used as received. Protein solutions were prepared by dissolution of a properly weighted amount of lysozyme in the corresponding solvent mixture. Both the added solvent and the final protein solution were filtered once over a $0.22 \mu \mathrm{m}$ Millex$\mathrm{GV}$ filter from Millipore $\left(\mathrm{H}_{2} \mathrm{O}\right.$ and $\mathrm{D}_{2} \mathrm{O}$ solvents and solutions) 
or a DMSO-safe Acrodisc syringe filter from Pall (all DMSOcontaining solvents and solutions) to remove dust and insoluble material. The protein concentrations were not corrected for potential loss during the filtration procedure. Unless otherwise stated, experiments have been carried out on samples containing $\leq 10 \mathrm{~g} \mathrm{~L}^{-1}$ lysozyme under salt-free conditions.

2.2. Methods. 2.1.1. Light Scattering. Light scattering measurements have been performed on a 3D cross-correlation instrument from LS-instruments equipped with a $\mathrm{HeNe}$ laser $(\lambda=623.8 \mathrm{~nm})$ and a Flex correlator. A refractive index matching bath of filtered decalin surrounded the cylindrical scattering cell, and the temperature was controlled at $20.0 \pm$ $0.1{ }^{\circ} \mathrm{C}$ using a Haake DC10-K20 thermostat.

The excess Rayleigh ratio, $\Delta R(\theta, C)$, was determined from static light scattering experiments using toluene as a reference according to

$$
\Delta R(\theta, C)=\frac{\left\langle I_{\text {sample }}\right\rangle-\left\langle I_{\text {solvent }}\right\rangle}{\left\langle I_{\text {toluene }}\right\rangle-\left\langle I_{\text {darkcurrent }}\right\rangle} R(\theta)_{\text {toluene }} \frac{n_{\text {sample }}^{2}}{n_{\text {toluene }}^{2}}
$$

with the scattering angle, $\theta$, the weight concentration of scattering objects, $C$, the corrected averaged scattered intensities of the sample, solvent, toluene, and blocked beam, $\left\langle I_{\text {sample }}\right\rangle$, $\left\langle I_{\text {solvent }}\right\rangle,\left\langle I_{\text {toluene }}\right\rangle$, and $\left\langle I_{\text {darkcurrent }}\right\rangle$, the Rayleigh ratio of toluene, $R(\theta)_{\text {toluene }}=1.34 \times 10^{5} \mathrm{~cm}^{-1}, 35$ and the refractive indices of the sample and the reference at $T=20.0{ }^{\circ} \mathrm{C}$ and $\lambda=632.8$ $\mathrm{nm}, n_{\text {sample }}$ and $n_{\text {toluene. }}$. Due to its small size $\left(R_{\mathrm{g}}=1.87 \mathrm{~nm} \ll\right.$ $\left.q^{-1}\right)$, lysozyme is a Rayleigh scatterer, such that

$$
\Delta R(\theta, C)=K C\left\langle M_{w}\right\rangle P(q) S(q)=K C\left\langle M_{w}\right\rangle S(q)
$$

with the averaged mass of the scatterers, $\left\langle M_{w}\right\rangle$, the form factor, $P(q)$, the structure factor, $S(q)$, and the magnitude of the scattering wave vector, $q$, given by

$$
q=\frac{4 \pi n}{\lambda_{0}} \sin \left(\frac{\theta}{2}\right)
$$

and the optical constant, $K$, given by

$$
K=\frac{4 \pi^{2} n^{2}}{N_{\mathrm{av}} \lambda^{4}}\left(\frac{\mathrm{d} n}{\mathrm{~d} C}\right)^{2}
$$

with Avogadro's number, $N_{\mathrm{av}}$, and the specific refractive index increment of the sample, $(\mathrm{d} n) /(\mathrm{d} C)$. The static structure factor at $\theta=90^{\circ}, S_{90}$, was calculated from eqs 2 and 4 using the measured $\Delta R\left(90^{\circ}, C\right), C=9.1-9.5 \mathrm{~g} \mathrm{l}^{-1}$ and $\left\langle M_{w}\right\rangle=14300 \mathrm{~g}$ $\mathrm{mol}^{-1}$.

As $q R_{\mathrm{g}} \ll 1$, the collective diffusion coefficient, $D_{\mathrm{c}}$, was determined from the initial decay of the autocorrelation function, $g_{1}(t)$, as determined from dynamic light scattering experiments at a fixed scattering angle of $90^{\circ}$ according to

$$
g_{2}(t)=A+B g_{1}^{2}(t)=A+B \mathrm{e}^{-2 t / \tau}=A+B \mathrm{e}^{-2 D_{c} q^{2} t}
$$

with the second (intensity) and first order (field) autocorrelation functions, $g_{2}(t)$ and $g_{1}(t)$, the delay time, $t$, the characteristic relaxation time, $\tau$, and two parameters $A$ and $B$ that equal unity in the case of a perfect experimental setup. Literature values of the hydrodynamic radius, $R_{\mathrm{h}}$, of native and denatured lysozyme were used to calculate the theoretical self-diffusion coefficient for each solvent composition from the Stokes-Einstein equation

$$
D_{0}=\frac{k T}{6 \pi \eta R_{\mathrm{h}}}
$$

with the Boltzmann constant, $k$, the temperature, $T$, and the solvent viscosity, $\eta$. The ratio between the static structure factor, $S_{90}$, and the hydrodynamic function, $H_{90}$, is then given by the ratio between the measured collective diffusion coefficient, $D_{\mathrm{c}}$, and the theoretical self-diffusion coefficient, $D_{0}$, according to ${ }^{36}$

$$
\frac{S_{90}}{H_{90}}=\frac{D_{0}}{D_{c}}
$$

2.1.2. Small Angle Neutron Scattering. Small angle neutron scattering experiments were performed at the Institut Max von Laue-Paul Langevin (ILL), Grenoble, France, on the D11 beamline and at the Paul Scherrer Institute, Villigen, Switzerland, on the SANS II beamline. At the ILL, we used circular 2 mm path length Hellma quartz cells and a thermostatted sample holder $\left(T=20^{\circ} \mathrm{C}\right)$. Two different sample-to-detector distances $(1.2$ and $8 \mathrm{~m})$ and collimation lengths $(5.5$ and $8 \mathrm{~m})$ at a fixed wavelength of $10 \AA$ were used to cover a $q$-range $0.02-0.3$ $\AA^{-1}$. At the PSI, we used rectangular $2 \mathrm{~mm}$ path length Hellma quartz cells and a thermostatted sample holder $\left(T=20^{\circ} \mathrm{C}\right)$. Two different sample-to-detector distances (1.2 and $4 \mathrm{~m})$ and collimation lengths ( 3 and $4 \mathrm{~m}$ ) at a fixed wavelength of $5.3 \AA$ were used to cover a $q$-range $0.02-0.3 \AA^{-1}$. The raw spectra were corrected for background scattering from the solvent, sample cell, and electronic noise by conventional procedures. The two-dimensional isotropic scattering spectra were corrected for detector efficiency by dividing with the incoherent scattering spectra of pure water, azimuthally averaged, and put to an absolute scale.

2.1.3. Attenuated Total Reflection-Infrared Spectroscopy. Infrared spectra of $7 \mathrm{~g} \mathrm{~L}^{-1}$ lysozyme solutions have been recorded with a Bomem Hartmann and Braun MB 155 FTIR spectrometer in attenuated total reflection (ATR) with a ZnSe crystal in a spectral region $600-4000 \mathrm{~cm}^{-1}$ using a resolution of $4 \mathrm{~cm}^{-1}$. The spectra presented are averages of 16 scans.

Circular Dichroism. Circular dichroism (CD) spectra were recorded on a Jasco J-715 spectropolarimeter. Lysozyme solutions of $0.04 \mathrm{~g} \mathrm{~L}^{-1}$ were scanned from 260 to $310 \mathrm{~nm}$ in a 1 $\mathrm{cm}$ quartz cell (near-ultraviolet range) using a data pitch of 0.1 $\mathrm{nm}$, a scan rate of $20 \mathrm{~nm} \mathrm{~min}{ }^{-1}$, and a bandwidth of $1 \mathrm{~nm}$. The spectra presented are averages of eight scans.

\section{Results and Discussion}

3.1. Protein-Protein Interactions. In aqueous DMSO solutions containing $\leq 10 \mathrm{~g} \mathrm{~L}^{-1}$ lysozyme without added salt, protein-protein interactions are dominated by long-range electrostatic repulsion. Lysozyme is strongly charged as the $\mathrm{pH}$ is far below the isoelectric point of $\sim 11$. Light scattering and small angle neutron scattering (SANS) experiments have been performed to probe these protein-protein interactions directly. We observe a significant depression of the forward scattering intensity due to repulsive interparticle interference effects for all DMSO volume fractions, $\varphi_{\text {DMSO }}$. Values for the structure 

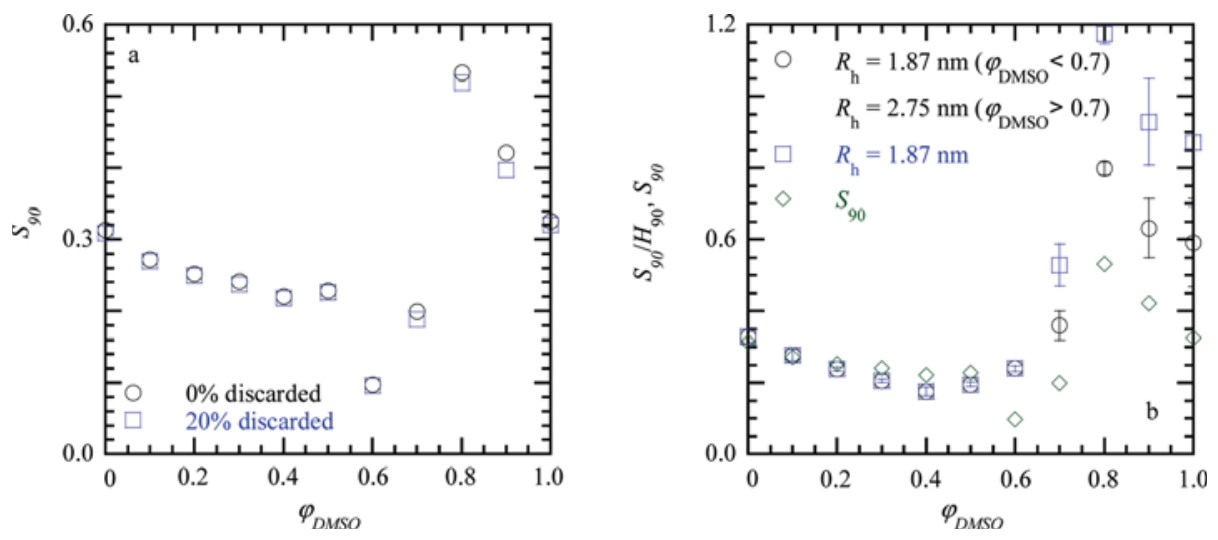

Figure 2. (a) Static structure factor, $S_{90}$, of $9.1-9.5 \mathrm{~g} \mathrm{~L}^{-1}$ lysozyme solutions in aqueous DMSO mixtures of $0 \leq \varphi_{\text {DMSO }} \leq 1$ measured at a scattering angle of $90^{\circ}$. A comparison between $S_{90}$ obtained upon including all measured intensity values $(O)$ and the $80 \%$ lowest intensity values $(\square)$ is given as a measure of the reliability of the experiment. (b) Comparison between the static structure factor, $S_{90}$, obtained from static light scattering experiments and the static structure factor divided by the hydrodynamic function, $S_{90} / H_{90}$, determined from dynamic light scattering experiments. The self-diffusion coefficient, $D_{0}$, was calculated from literature values of the hydrodynamic radius, $R_{\mathrm{h}}$ of ( $\square$ ) the native protein for $0 \leq \varphi_{\text {DMSO }} \leq 1$ and of $(O)$ the native protein for $0 \leq \varphi_{\text {DMSO }} \leq 0.6\left(R_{\mathrm{h}}=1.87\right)$ and the denatured protein for $0.7 \leq \varphi_{\text {DMSO }} \leq 1.0\left(R_{\mathrm{h}} \sim 2.75\right) .{ }^{37,41,43}$

TABLE 1: Results of an Inverse Fourier Transform Analysis of the Small Angle Neutron Scattering Curves Depicted in Figure $4^{a}$

\begin{tabular}{cccccccc}
\hline$\varphi_{\text {DMSO }}$ & $C_{\mathrm{LYS}} / \mathrm{g} \mathrm{L}^{-1}$ & $R_{\mathrm{g}} / \mathrm{nm}$ & $I(0) / \mathrm{cm}^{-1}$ & $\Delta \rho^{b} / 10^{10} \mathrm{~cm}^{-2}$ & $\bar{v}^{c} / \mathrm{cm}^{3} \mathrm{~g}^{-1}$ & $M / \mathrm{kg} \mathrm{mol}^{-1}$ & $P_{\text {agg }}$ \\
\hline 0 & 28.50 & $1.38 \pm 0.09$ & $0.35 \pm 0.01$ & 3.29 & 0.712 & $13.3 \pm 0.3$ & $0.95 \pm 0.02$ \\
0.3 & 27.73 & $1.30 \pm 0.03$ & $0.31 \pm 0.01$ & 2.96 & 0.714 & $14.9 \pm 0.6$ & $1.04 \pm 0.04$ \\
0.5 & 27.54 & $1.29 \pm 0.03$ & $0.31 \pm 0.01$ & 2.74 & 0.715 & $17.9 \pm 0.8$ & $1.24 \pm 0.06$ \\
0.6 & 27.73 & $1.28 \pm 0.03$ & $0.26 \pm 0.01$ & 2.62 & 0.720 & $16.1 \pm 0.7$ & $1.13 \pm 0.05$ \\
0.7 & 9.90 & $1.81 \pm 0.03$ & $0.066 \pm 0.00$ & 2.10 & 0.730 & $17.1 \pm 0.5$ & $1.20 \pm 0.04$ \\
0.8 & 9.57 & $2.48 \pm 0.03$ & $0.055 \pm 0.00$ & 1.99 & 0.734 & $16.2 \pm 0.3$ & $1.14 \pm 0.02$ \\
0.9 & 9.47 & $2.44 \pm 0.06$ & $0.044 \pm 0.01$ & 1.88 & 0.711 & $15.7 \pm 1.8$ & $1.15 \pm 0.13$ \\
1.0 & 9.17 & $2.37 \pm 0.02$ & $0.091 \pm 0.00$ & 3.32 & 0.705 & $10.8 \pm 0.5$ & $0.79 \pm 0.04$
\end{tabular}

${ }^{a}$ The listed values are mean values and corresponding standard deviations as determined for three different incoherent background subtractions (see Supporting Information for more details). ${ }^{b}$ The scattering length density of lysozyme is calculated in accordance with the analysis reported in ref 54. We assume that lysozyme has 193 exchangeable protons in the folded states and 263 exchangeable protons in the unfolded states. In all solvents but pure $d_{6}$-DMSO, we assume that all hydrogens on lysozyme will have been exchanged by deuterium (the excess of deuterium is $>80$-fold), such that the scattering length density of lysozyme is $3.08 \times 10^{10} \mathrm{~cm}^{-2}$ for $0<\varphi_{\text {DMSO }}<0.6,3.49 \times 10^{10}$ $\mathrm{cm}^{-2}$ for $0.7<\varphi_{\text {DMSO }}<0.9$, and $1.93 \times 10^{10} \mathrm{~cm}^{-2}$ for $\varphi_{\text {DMSO }}=1.0$. Note that potential variations in protein hydration ${ }^{51}$ as a function of solvent composition have not been taken into account. ${ }^{c}$ The values for the specific volume are taken from ref 55 covering the largest range of DMSO volume fractions. Slightly different values were reported by others for various values of $\varphi_{\text {DMSO }}{ }^{29}$

factor in the zero- $q$ limit as determined from static (SLS) and dynamic light scattering (DLS) experiments were found to be well below 1 for all values of $\varphi_{\text {DMSO }}$ (Figure 2). As will be discussed later on in this Article, this is confirmed by the small angle neutron scattering experiments. The static structure factor, $S_{90}$, as obtained from static light scattering and the quotient of the static structure factor and the hydrodynamic function, $S_{90} / H_{90}$, as obtained from dynamic light scattering are coincident up to $\varphi_{\text {DMSO }} \sim 0.6$, after which $S_{90} / H_{90}>S_{90}$. To calculate $S_{90} / H_{90}$ for $\varphi_{\text {DMSO }}>0.6$ from the theoretical self-diffusion coefficient, $D_{0}$ based on eq 7 , we assumed that the hydrodynamic radius of DMSO-denatured lysozyme lies somewhere between the values reported for native lysozyme $\left(R_{\mathrm{h}}=1.87 \mathrm{~nm}\right)$ and lysozyme under strong denaturing conditions $\left.\left(2.18 \leq R_{\mathrm{h}} \leq 2.75 \mathrm{~nm}\right)\right)^{37-41}$ Both assumptions lead to $S_{90} / H_{90}>S_{90}$ for $\varphi_{\text {DMSO }} \sim 0.6$; i.e., $H_{90}<1$ indicative of repulsive hydrodynamic interactions. We observe three distinct regimes of protein-protein interactions: in regimes I $\left(0 \leq \varphi_{\text {DMSO }}<0.6\right)$ and III $\left(0.8<\varphi_{\text {DMSO }} \leq 1.0\right)$ the overall interparticle repulsion $\left(S_{90}\right)$ increases upon increasing $\varphi_{\text {DMSO }}$, whereas in regime II $\left(0.6 \leq \varphi_{\text {DMSO }} \leq 0.8\right)$, protein- protein interactions become more attractive, but remain repulsive, upon increasing $\varphi_{\text {DMSO }}$. We tentatively attribute this complex nonmonotonous behavior to the interplay between exposure of different amino acids due to the DMSO-induced structural transitions and the decrease in the dielectric permittivity of the solvent from 78.36 to 47.0 upon increasing $\varphi_{\text {DMSO }}{ }^{42}$ The overall repulsive character of the protein-protein interactions and the low lysozyme concentrations, $C_{\mathrm{LYS}}<10 \mathrm{~g} \mathrm{~L}^{-1}$, explain why

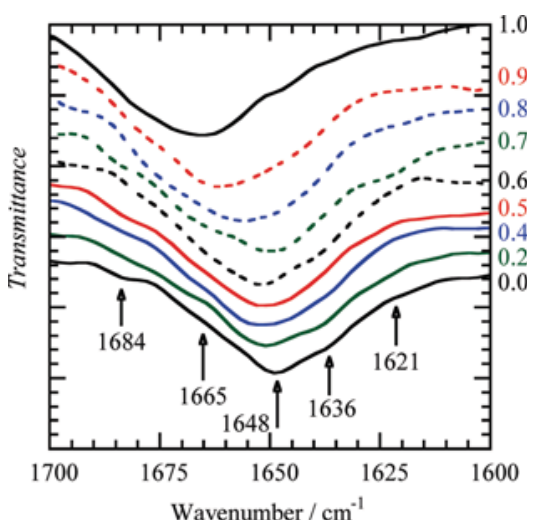

Figure 3. ATR-IR spectra in the amide I region of $7 \mathrm{~g} \mathrm{~L}^{-1}$ lysozyme solutions in $\mathrm{D}_{2} \mathrm{O} / d_{6}$-DMSO solutions containing $0.0 \leq \varphi_{\text {DMSO }} \leq 1.0$ as indicated. The arrows indicate the positions of some characteristic bands: $1684 \mathrm{~cm}^{-1}$ (turns), $1665 \mathrm{~cm}^{-1}$ (free carbonyl groups, i.e. not H-bonded to other amino acids), $1648 \mathrm{~cm}^{-1}$ ( $\alpha$-helix), $1636 \mathrm{~cm}^{-1}$ (intramolecular $\beta$-sheet), $1622 \mathrm{~cm}^{-1}$ (intermolecular $\beta$-sheet). For presentation purposes the spectra have been shifted in the vertical direction. 

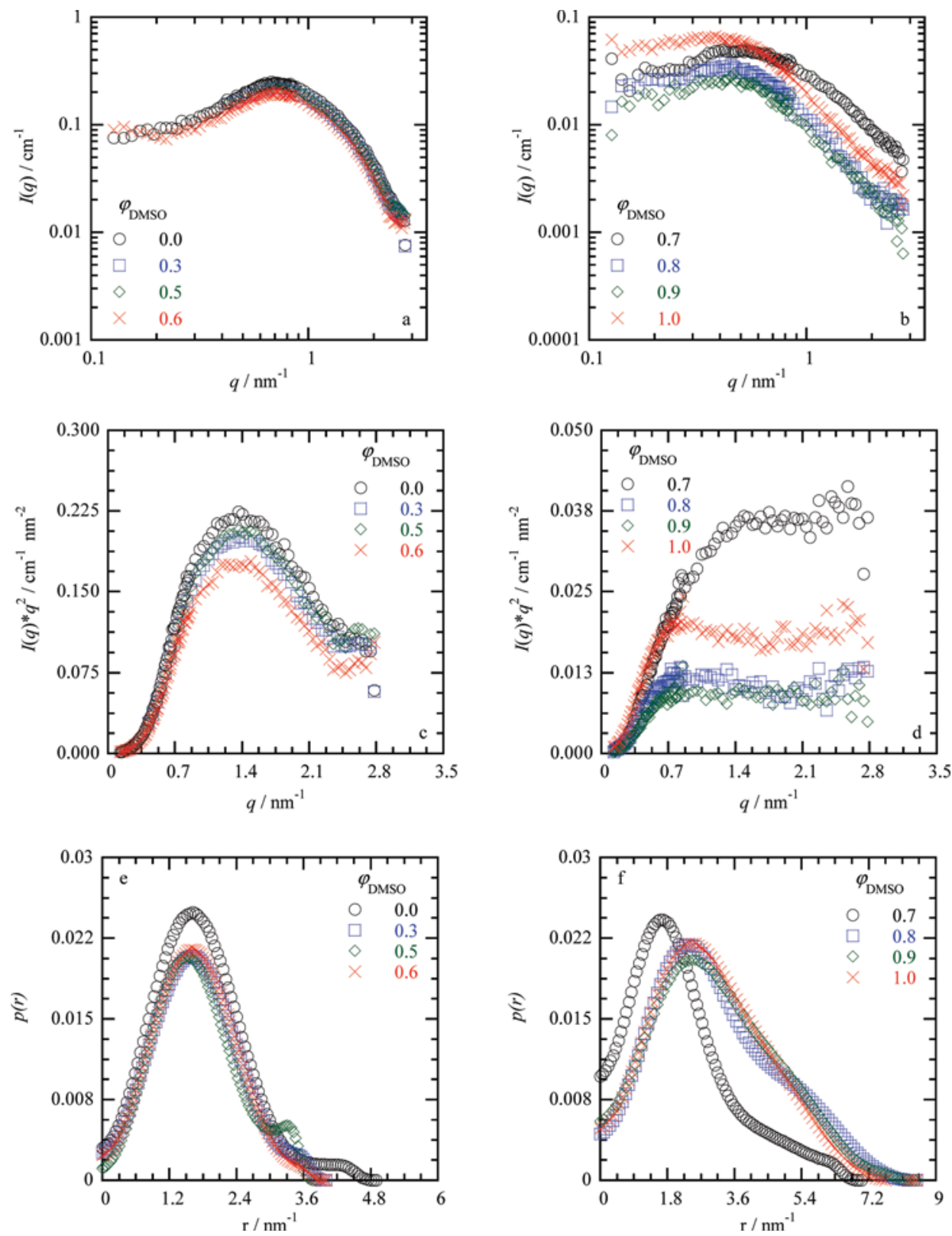

Figure 4. Small angle neutron scattering profiles $(I(q)$ versus $q)$, Kratky representations $\left(I(q) \cdot q^{2}\right.$ versus $\left.q\right)$, and pair-distance-distribution functions $(p(r))$ for $(\mathrm{a}, \mathrm{c}, \mathrm{e}) 30 \mathrm{~g} \mathrm{~L}^{-1}$ lysozyme solutions at $\varphi_{\text {DMSO }} \leq 0.6$ and $(\mathrm{b}, \mathrm{d}, \mathrm{f}) 10 \mathrm{~g} \mathrm{~L}^{-1}$ lysozyme solutions at $\varphi_{\text {DMso }} \geq 0.7$. Note that in parts e and $\mathrm{f}$, the pair-distance-distribution functions have been normalized to a total area of 1 ; i.e., the curves should superimpose in case particle shape and structure are independent of $\varphi_{\text {DMSO }}$.

lysozyme does not aggregate even under conditions where the protein is denatured (see also Table 1).

ATR-IR experiments on $7 \mathrm{~g} \mathrm{~L}^{-1}$ lysozyme solutions confirm the absence of aggregation, as the $\sim 1621 \mathrm{~cm}^{-1}$ peak which is commonly associated with intermolecular $\beta$-sheet formation, is absent (Figure 3). In an upcoming paper, we will demonstrate that the signal at $1621 \mathrm{~cm}^{-1}$ appears for $0.7 \leq \varphi_{\text {DMSO }} \leq 0.9$ at protein concentrations in excess of $10 \mathrm{~g} \mathrm{~L}^{-1}$, in accordance with ref 30 . We therefore conclude that the critical aggregation concentration for $0.7 \leq \varphi_{\text {DMSO }} \leq 0.9$ lies somewhere between 10 and $20 \mathrm{~g} \mathrm{~L}^{-1}$.

3.2. Global Structure. DMSO-induced changes in lysozyme size and shape were investigated in SANS experiments for lysozyme concentrations below the critical aggregation concentration, corresponding to $C_{\mathrm{LYS}} \sim 30 \mathrm{~g} \mathrm{~L}^{-1}$ for $0 \leq \varphi_{\text {DMSO }} \leq$ 0.6 and $C_{\mathrm{LYS}} \sim 10 \mathrm{~g} \mathrm{~L}^{-1}$ for $0.7 \leq \varphi_{\text {DMSO }} \leq 1.0$. The scattering profiles obtained for $\varphi_{\text {DMSO }} \leq 0.6$ resemble each other but clearly differ from those obtained for higher $\varphi_{\text {DMSO }}($ Figure 4$)$. For $\varphi_{\text {DMSO }} \leq 0.6$, the scattered intensity, $I(q)$, decays proportional to $q^{-4}$ in the high $q$-limit, whereas $I(q) \propto q^{-2}$ for $\varphi_{\text {DMSO }}$ $\geq 0.7$. As a consequence, we observe a peak in the Kratky representations of the data for $\varphi_{\text {DMSO }} \leq 0.6$ and a plateau for $\varphi_{\text {DMSO }} \geq 0.7$, in accordance with Porod-scattering of homogeneous spheres with a smooth surface and random coil polymers, respectively (Figure 4c,d). In accordance with differential scanning calorimetry experiments, ${ }^{20-23}$ we find that the folding-unfolding transition occurs at $\varphi_{\text {DMSO }} \sim 0.7$.

An inverse Fourier transform analysis (IFT) results in somewhat unconventional pair-distance-distribution functions, $p(r)^{44,45}$ (Figure 4e,f), as lysozyme (particularly in the denaturated states) does not resemble a homogeneous particle with a smooth surface. As a consequence, we find that $p(r) \neq 0$ at $r=$ 0 . In the Supporting Information, we demonstrate that this condition can only be fulfilled by oversubtraction of the 
background. We also demonstrate that the Kratky representations are highly sensitive to the background subtraction (see Supporting Information), while the gyration radii and zero- $q$ intensity values are not (Table 1 ). The radii of gyration, $R_{\mathrm{g}},{ }^{44}$ were extracted through an IFT analysis according to

$$
R_{\mathrm{g}}^{2}=\frac{1}{2} \frac{\int_{0}^{D_{\max }} p(r) r^{2} \mathrm{~d} r}{\int_{0}^{D_{\max }} p(r) \mathrm{d} r}
$$

The values have been listed in Table 1 along with the molecular weight of scatters that can be determined from the data (as they have been put to absolute scale), ${ }^{46}$ according to

$$
M_{\mathrm{SANS}}=I(0) \frac{N_{\mathrm{av}}}{c \Delta \rho_{\mathrm{M}}^{2}}
$$

with the molecular weight, $M_{\mathrm{SANS}}$, the forward scattering intensity at $q=0, I(0) / \mathrm{cm}^{-1}$, the concentration, $\mathrm{c} / \mathrm{g} \mathrm{cm} \mathrm{cm}^{-3}$, Avogadro's number, $N_{\mathrm{av}}$, and the scattering length density difference per mass, $\Delta \rho_{\mathrm{M}} / \mathrm{cm} \mathrm{g}^{-1}$, being

$$
\Delta \rho_{\mathrm{M}}=\Delta \rho \bar{v}
$$

with the scattering length density difference, $\Delta \rho / \mathrm{cm}^{-2}$, and the specific volume of the scatterer in solution, $\bar{v} / \mathrm{cm}^{3} \mathrm{~g}^{-1}$. The results confirm the absence of any significant amount of particle aggregation, as the values of the molecular weight and aggregation numbers (determined from $M_{\mathrm{SANS}} / M_{\mathrm{LZ}}$ with $M_{\mathrm{LZ}}=14300$ $\mathrm{g} \mathrm{mol}^{-1}$ ) correspond to those for single protein macromolecules. The fluctuations in $M_{\mathrm{SANS}} / M_{\mathrm{LZ}}$ (up to $20 \%$ ) reflect uncertainties in the calculation of the scattering length density, $\Delta \rho$, due to uncertainties in the amount of exchangeable protons, water impurities in the hygroscopic solvent $d_{6}$-DMSO, and the protein specific volume. For $\varphi_{\text {DMSO }} \leq 0.6$, we obtain radii of gyration that are in excellent agreement with those reported by others ${ }^{32,47-51}$ and the value of $R_{\mathrm{g}}=1.4 \mathrm{~nm}$ as calculated from the crystal structure, ${ }^{47}$ while for $\varphi_{\text {DMSO }} \geq 0.8$ the values nicely correspond to those reported for lysozyme under a variety of experimental conditions leading to protein unfolding. ${ }^{32,37,50-53}$ Note that the radius of gyration for the ideal random coil state is expected to be $>3.5 \mathrm{~nm}$, i.e., far larger than the values observed here. ${ }^{51}$

From the above, it becomes clear that, apparently, we cannot distinguish between the "unfolded" $\left(0.7<\varphi_{\text {DMSO }}<0.8\right)$ and "partially collapsed" $\left(0.9<\varphi_{\text {DMSO }}<1.0\right)$ regimes based on the overall particle shape (Figure 4), but we can do so based on the particle-particle interactions and gyration radii (Figure 5). In the unfolded regime, the interparticle interactions are least repulsive as it corresponds to maximum solvent exposure of the (majority of) amino acids inducing additional attraction between proteins as compared to the folded and partially collapsed regime. At the same time, we observe a large increase in the radius of gyration upon unfolding and a small, but noticeable, decrease of the radius of gyration in the partially collapsed regime. Note that the protein is more swollen in the partially collapsed regime than in the folded regime and larger than would be expected for a synthetic polymer in poor solvent conditions. Tentatively, we attribute this finding to conformational restrictions imposed by the four intact disulfide bonds, which serve to stabilize the native structure and prevent a full DMSO-induced structural reconfiguration. Moreover, we note

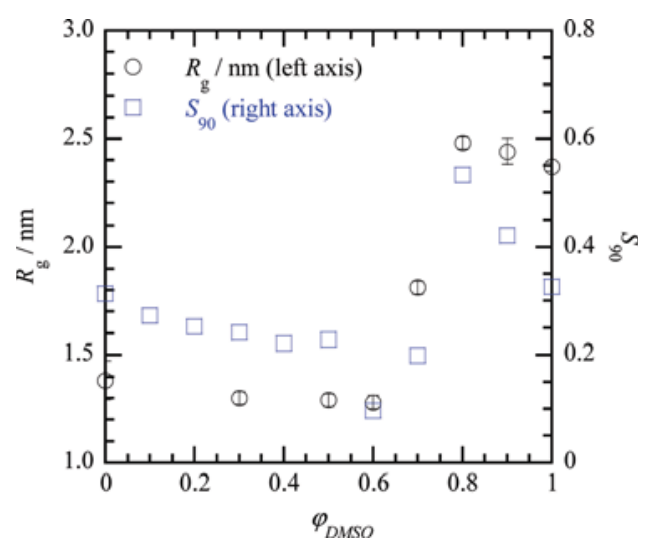

Figure 5. ( $\square$ ) Static structure factor, $S_{90}$, of $9.1-9.5 \mathrm{~g} \mathrm{~L}^{-1}$ and $(\bigcirc)$ gyration radius, $R_{\mathrm{g}}$, of $9.2-28.5 \mathrm{~g} \mathrm{~L}^{-1}$ lysozyme in aqueous DMSO mixtures at $0 \leq \varphi_{\text {DMSO }} \leq 1$ as determined from static light scattering and small angle neutron scattering experiments (see also Figure 2 and Table 1).

that a protein is a heteropolymer consisting of many different monomers (amino acids) and not a homopolymer which consists of one type of monomer, such that some amino acids, typically abundant close to the protein's surface, are in good solvent conditions and some are in bad solvent conditions.

3.3. Local Structure. 3.3.1. Tertiary Structure. Having established that, at ambient temperatures, lysozyme is predominantly present in a folded state for $\varphi_{\text {DMSO }} \leq 0.6$, while it is predominantly unfolded for higher values of $\varphi_{\text {DMSO }}$, we turn our attention to some spectroscopic experiments that probe the local environment of specific residues, such as tryptophan (Trp). The characteristic tertiary structure of lysozyme in aqueous DMSO mixtures can be monitored with near-UV circular dichroism (CD) experiments (Figure 6). ${ }^{27,28}$ The spectra at DMSO volume fractions up to $\varphi_{\text {DMSO }}=0.6$ resemble each other, while they are clearly different from the spectra for $\varphi_{\text {DMSO }} \geq$ 0.7. If we monitor the CD signal at a fixed wavelength as a function of $\varphi_{\text {DMSO }}$, we see that the signal is enhanced up to $\varphi_{\text {DMSO }} \sim 0.6$, while it strongly decreases and eventually disappears at higher DMSO volume fractions. The environment of the tryptophan residues in lysozyme compacts up to $\varphi_{\text {DMSO }}$ $=0.6$, which results in enhanced stability with respect to thermal denaturation up to $\varphi_{\text {DMSO }}=0.4,{ }^{22}$ while the protein remains predominantly folded at ambient temperatures. When the characteristic tertiary structure is completely lost $\left(\varphi_{\text {DMSO }}>0.7\right)$, the protein is either in an "unfolded" (regime II) or "partially collapsed" (regime III) conformation having a larger $R_{\mathrm{g}}$ than the folded conformation (regime I). The observed compaction at low $\varphi_{\text {DMSO }}$ may seem unexpected in light of the propensity of DMSO to break intramolecular $\mathrm{H}$-bonds that are essential for the protein's secondary and tertiary structure. However, one has to realize that DMSO acts in a number of different ways and, more importantly, that its effect may be heterogeneous within the protein: while some regions compact, others may become more flexible. Indeed, a reduction in the chemical shift dispersion in nuclear magnetic resonance (NMR) spectra of lysozyme in $\varphi_{\text {DMSO }}=0.5,0.8,0.9$, and 1.0 indicates that several regions in the protein are more flexible as compared to that of the protein in $\varphi_{\text {DMSO }}=0 .{ }^{1,27,28} \mathrm{We}$ might speculate that DMSO does not unfold lysozyme, even though many H-bonds may be broken and some parts of the macromolecule are more flexible, until the stability of the region around the buried tryptophan residues is compromised. The UV-vis experiments of Hamaguchi et al. support this idea. At the folding-unfolding transition corresponding to $\varphi_{\text {DMSO }} \sim 0.7$, the refractive index of the 

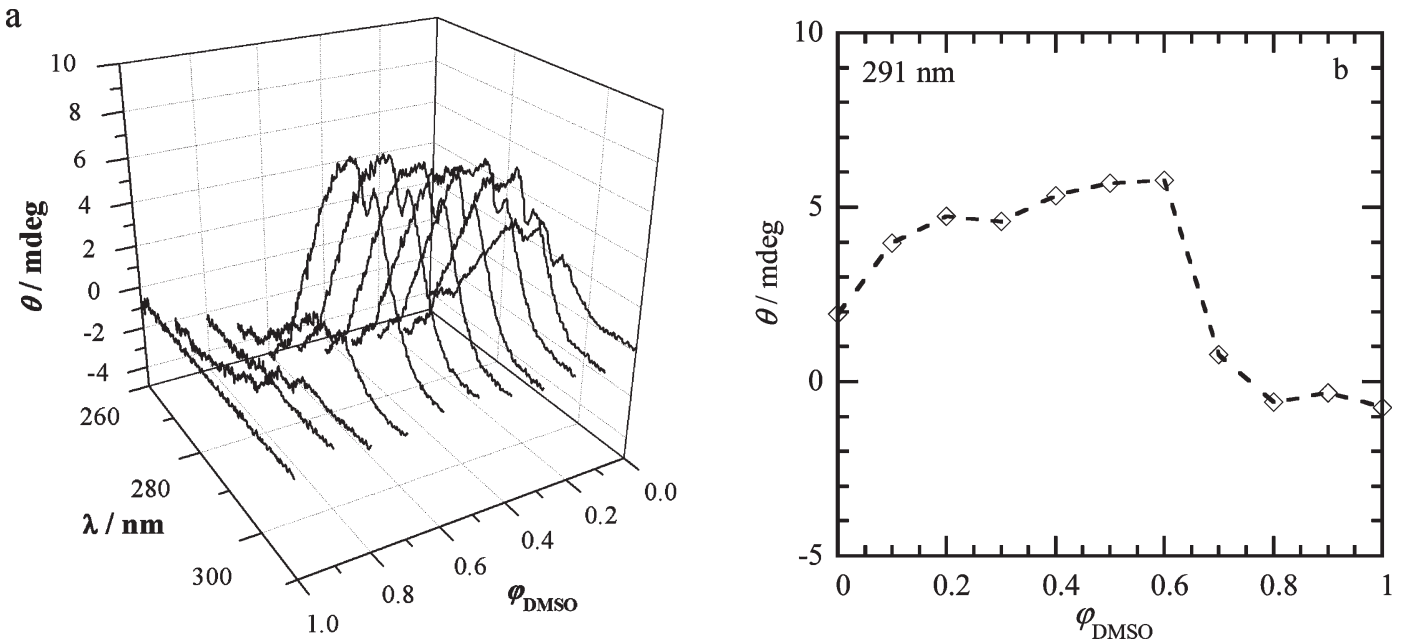

Figure 6. (a) CD spectra obtained from near-UV circular dichroism experiments on $0.04 \mathrm{~g} \mathrm{~L}^{-1}(3 \mu \mathrm{M})$ lysozyme in aqueous DMSO mixtures of $0<\varphi_{\text {DMSO }}<1.0$. (b) The CD signal at $\lambda=291 \mathrm{~nm}$ as a function of $\varphi_{\text {DMSO }}$.

lysozyme interior surrounding the buried tryptophan residues was found to be close to that of the binary solvent mixture; i.e., $n_{\text {interior }} \sim n_{0.7}=1.440 .{ }^{29}$ Thus, the solvent environment has become similar to that of the protein's interior at $\varphi_{\text {DMSO }} \sim 0.7$, such that there is no enthalpic gain associated with burying residues deep within the protein and, if sufficient $\mathrm{H}$-bonds are broken, the protein will unfold due to entropic reasons. ${ }^{29}$

3.3.2. Secondary Structure. In the above, we have documented a tendency toward unfolding upon increasing DMSO volume fraction which has to be accompanied by a sufficient decrease in the number of intramolecular H-bonds between side groups responsible for the protein's tertiary structure. Intramolecular $\mathrm{H}$-bonds between backbone elements are connected to the secondary structural elements such as $\alpha$-helices, $\beta$-sheets, and turns. If likewise broken, we should observe a decrease in these characteristic structural elements and an increase in the percentage of "random coil"-type features upon increasing DMSO volume fraction.

Areas et al. have employed Raman spectroscopy to investigate the secondary structure of lysozyme in $72 \mathrm{~g} \mathrm{~L}^{-1}$ aqueous DMSO mixtures (well above the critical aggregation concentration for $\varphi_{\text {DMSO }}=0.75$ and 0.9 ) in a quantitative manner. ${ }^{1}$ The results show a nearly constant percentage of $\mathrm{H}$-bond mediated secondary structural elements $(70-75 \%)$ up to $\varphi_{\text {DMSO }}=0.75$, while their relative abundance is strongly $\varphi_{\text {DMSO }}$ dependent: the $\alpha$-helix content decreases and the $\beta$-sheet content increases toward $\varphi_{\text {DMSO }}=0.75$. At higher volume fractions, the summed $\alpha$-helix and $\beta$-sheet content decreases, while peak positions deviate from orthodox assignments: only "loose" or "molten" secondary structural elements can be observed as more and more H-bonds between backbone amino acid residues break. Fourier transform infrared spectroscopy yields similar results (see for example Figure 3 and ref 30). Obviously, the macromolecule loses a significant amount of its globular, compact character at high DMSO volume fractions and exhibits random coil statistics (Figures 4), but it is not fully unstructured: some secondary structural elements are retained. Recent experimental works as well as MD simulations on other protein systems have also provided some evidence for the persistence of secondary structural elements in the denatured state. ${ }^{51,56-60}$ Apparently, the tertiary structure is lost well before all (nonorthodox) secondary structure is lost.

\section{Conclusions}

We have investigated the size, shape, structure, and interactions of the globular protein lysozyme in the ternary system lysozyme/DMSO/water at low protein concentrations where aggregation is suppressed due to overall repulsive interactions. Three structural regimes have been identified: the "folded" $(0$ $\left.<\varphi_{\text {DMSO }}<0.7\right)$, "unfolded" $\left(0.7 \leq \varphi_{\text {DMSO }}<0.9\right)$, and "partially collapsed" $\left(0.9 \leq \varphi_{\text {DMSO }}<1.0\right)$ regimes, respectively. Lysozyme adopts an overall folded state with an average radius of gyration of $1.3 \pm 0.1 \mathrm{~nm}$ for $\varphi_{\text {DMSO }}<0.6$, and unfolds into a particle with an average $R_{\mathrm{g}}$ of $2.4 \pm 0.1 \mathrm{~nm}$ for $\varphi_{\text {DMSO }}>0.7$. This drastic change in the protein's size, shape, and tertiary structure is preceded by a compaction of the local environment of the tryptophan residues and accompanied by a large increase in the protein's overall flexibility. In terms of secondary structure, there is a gradual loss of $\alpha$-helix and concomitant increase of $\beta$-sheet structural elements toward $\varphi_{\text {DMSO }}=0.7$, while an increase in $\varphi_{\text {DMSO }}$ beyond this point finally reduces the amount of secondary structural elements. Still, the protein does not adopt a fully random coil structure: some secondary structural elements remain in the unfolded state when the characteristic tertiary structure has already been lost. We have attempted to relate these structural changes to the important physical mechanisms that come into play: the solvent microstructure is strongly dependent on the DMSO volume fraction, DMSO acts as strong $\mathrm{H}$-bond acceptor, and DMSO is a bad solvent for the protein backbone and a number of relatively polar side groups, but a good solvent for relatively apolar side groups, such as tryptophan.

Establishing a detailed understanding of the structural changes that lysozyme undergoes upon increasing $\varphi_{\text {DMSO }}$ in the ternary system lysozyme/DMSO/water is an essential first step in our attempt to understand the physical nature of the sol-gel transition in these systems at higher lysozyme volume fractions. ${ }^{2}$ The conformational transitions observed at lower volume fractions give rise to a complicated nonmonotonous dependence of protein - protein interactions upon increasing $\varphi_{\text {DMSO }}$, which plays a decisive role in the sol-gel transition, possibly resulting in a dependence of the gel microstructure on $\varphi_{\text {DMSO }}$. Eventually, we hope that our insights will reveal pathways to the formation of active gels (that is, fractal gels or glasses of globular lysozyme macromolecules with an intact catalytic cavity such that its enzymatic activity is preserved) and high quality diffraction crystals of (partially) unfolded lysozyme in order to study the 
folding-unfolding transition in detail. Although the universality of the results for other proteins and organic solvents remains to be proven, we would like to note that several other ternary systems display similar features (see for examples refs 31-33). We hope that our understanding of this particular system can serve as a basis for a detailed understanding of conformational changes in a great number of other protein/organic solvent/water systems. We would like to stress the implications for the use of DMSO in biomedical applications, which should be limited to very small dosages and certainly accompanied by appropriate toxicity assays. Finally, we would like to point out that the addition of an organic solvent to a protein solution can, in some cases, be an efficient way of shifting the balance between conformational states such that the link between protein structure and aggregation/gelation may be studied. This approach may be fruitful in studies of, for example, the potential existence of aggregation prone conformational states, which could provide valuable information in the fields of medicine and food science. In the former, there may be a need to prevent oligomerization and fibrillation events which are associated with a number of neurodegenerative diseases, whereas in food science there exists a desire to control the number and structure of aggregates to achieve a solid phase, such as a gel, with desired properties in terms of texture, stiffness, and transparency. Future work, in particular single molecule studies and molecular dynamic simulations with explicit solvent, might address the presumed structural heterogeneity of the various (un)folded states.

Acknowledgment. The small angle neutron scattering experiments were performed at the Swiss spallation neutron source SINQ, Paul Scherrer Institute, Villigen, Switzerland and the Institut Max von Laue-Paul Langevin (ILL), Grenoble, France. We are grateful for the neutron beam time and we acknowledge the help of our local contact at the SANS II beamline L. Almasy. We gratefully acknowledge a financial contribution from the Adolphe Merkle Foundation. I.K.V. is supported by an European Marie Curie Fellowship (FP7-PEOPLE-IEF-2008, Contract 236723). E.P.G.A. acknowledges support from FAPESP (Brazil). W.A.C. was recipient of an undergraduate scholarship from FAPESP and is grateful to IQUSP (Brazil) and the Adolphe Merkle Foundation for supporting a two-month research period at University of Fribourg, Switzerland.

Supporting Information Available: Information on the effect of background subtraction on the small angle neutron scattering profiles depicted in Figure 4.

\section{References and Notes}

(1) Areas, E. P. G.; Areas, J. A. G.; Hamburger, J.; Peticolas, W. L.; Santos, P. S. On the high viscosity of aqueous solution of lysozyme induced by some organic solvents. J. Colloid Interface Sci. 1996, 180, 578-589.

(2) Areas, E. P. G.; Cruz, W. A. Protein-polysaccharide viscoelastic matrices: synergic effects of amylose on lysozyme physical gelation in aqueous dimethylsulfoxide. Soft Matter 2009, 5, 4240-4249.

(3) Gordeliy, V. I.; Kiselev, M. A.; Lesieur, P.; Pole, A. V.; Teixeira, J. Lipid membrane structure and interactions in dimethyl sulfoxide/water mixtures. Biophys. J. 1998, 75, 2343-2351.

(4) Lovelock, J. E.; Bishop, M. W. H. Prevention of freezing damage to living cells by dimethyl sulphoxide. Nature 1959, 183, 1394-1395.

(5) Yu, Z.-W.; Quinn, P. J. Dimethyl sulphoxide: a review of applications in cell biology. Biosci. Rep. 1994, 14, 259-281.

(6) Williams, A. C.; Barry, B. W. Penetration enhancers. Adv. Drug Delivery Rev. 2004, 56, 603-618.

(7) Jacob, S. W. In Pharmacology of DMSO. Dimethylsulphoxide; Jacob, S. W., Rosenbaum, E. E., Wood, D. C., Eds.; Marcel and Dekker: New York, 1971; Vol. 1.

(8) Schmidt, B.; Ho, L.; Hogg, P. J. Allosteric disulfide bonds. Biochemistry 2006, 45, 7429-7433.
(9) Abkevich, V. I.; Shakhnovich, E. I. What can disulfide bonds tell us about protein energetics, function and folding: Simulations and bioninformatics analysis. J. Mol. Biol. 2000, 300, 975-985.

(10) Yokota, A.; Izutani, K.; Takai, M.; Kubo, Y.; Noda, Y.; Koumoto, Y.; Tachibana, H.; Segawa, S. The transition state in the folding-unfolding reaction of four species of three-disulfide variant of hen lysozyme: The role of each disulfide bridge. J. Mol. Biol. 2000, 295, 1275-1288.

(11) Areas, E. P. G.; Menezes, H. H. A.; Santos, P. S.; Areas, J. A. G. Hydrodynamic, optical and spectroscopic studies of some organic-aqueous binary systems. J. Mol. Liq. 1999, 79, 45-58.

(12) Soper, A. K.; Luzar, A. A neutron-diffraction study of dimethylsulfoxide water mixtures. J. Chem. Phys. 1992, 97, 1320-1331.

(13) Rasmussen, D. H.; MacKenzie, A. P. Phase diagram for system water-dimethylsulphoxide. Nature 1968, 220, 1315-1317.

(14) Markarian, S. A.; Terzyan, A. M. Surface tension and refractive index of dialkylsulfoxide plus water mixtures at several temperatures. J. Chem. Eng. Data 2007, 52, 1704-1709.

(15) Lam, S. Y.; Benoit, R. L. Some thermodynamic properties of dimethylsulfoxide-water and propylene carbonate-water systems at 25 degrees C. Can. J. Chem. 1974, 52, 718-722.

(16) Shin, D. N.; Wijnen, J. W.; Engberts, J.; Wakisaka, A. On the origin of microheterogeneity: A mass spectrometric study of dimethyl sulfoxidewater binary mixture. J. Phys. Chem. B 2001, 105, 6759-6762.

(17) Shin, D. N.; Wijnen, J. W.; Engberts, J.; Wakisaka, A. On the origin of microheterogeneity: Mass spectrometric studies of acetonitrile-water and dimethyl sulfoxide-water binary mixtures (part 2). J. Phys. Chem. B 2002, 106, 6014-6020.

(18) da Silva, M. A.; El Seoud, O. A.; Areas, E. P. G. Lysozyme gelation in mixtures of tetramethylurea with protic solvents: Use of solvatochromic indicators to probe medium microstructure and solute-solvent interactions. J. Mol. Struct. 2007, 841, 51-60.

(19) Arakawa, T.; Kita, Y.; Timasheff, S. N. Protein precipitation and denaturation by dimethyl sulfoxide. Biophys. Chem. 2007, 131, 62-70.

(20) Fujita, Y.; Izumiguchi, S.; Noda, Y. Effect of dimethylsulfoxide and its homologs on the thermal-denaturation of lysozyme as measured by differential scanning calorimetry. Int. J. Pept. Protein Res. 1982, 19, 2531.

(21) Kamiyama, T.; Liu, H. L.; Kimura, T. Preferential solvation of lysozyme by dimethyl sulfoxide in binary solutions of water and dimethyl sulfoxide. J. Therm. Anal. Calorim. 2009, 95, 353-359.

(22) Torreggiani, A.; Di Foggia, M.; Manco, I.; De Maio, A.; Markarian, S. A.; Bonora, S. Effect of sulfoxides on the thermal denaturation of hen lysozyme: A calorimetric and Raman study. J. Mol. Struct. 2008, 891, 115122.

(23) Lai, B.; Cao, A. N.; Lai, L. H. Organic cosolvents and hen egg white lysozyme folding. Biochim. Biophys. Acta, Protein Struct. Mol. 2000, $1543,115-122$.

(24) Flory, P. J. Principles of Polymer Chemistry; Cornell University Press: Ithaca, NY, 1953

(25) Degennes, P. G. Collapse of a polymer-chain in poor solvents. $J$. Phys., Lett. 1975, 36, L55-L57.

(26) Stockmayer, W. H. Problems of the statistical thermodynamics of dilute polymer solutions. Makromol. Chem. 1960, 35, 54-74.

(27) Bhattacharjya, S.; Balaram, P. Effects of organic solvents on protein structures: Observation of a structured helical core in hen egg-white lysozyme in aqueous dimethylsulfoxide. Proteins 1997, 29, 492-507.

(28) Knubovets, T.; Osterhout, J. J.; Klibanov, A. M. Structure of lysozyme dissolved in neat organic solvents as assessed by NMR and CD spectroscopies. Biotechnol. Bioeng. 1999, 63, 242-248.

(29) Hamaguchi, K. Structure of muramidase (lysozyme): VIII Effect of dimethyl sulfoxide on the stability of muramidase. J. Biochem. 1964, $56,441-449$.

(30) Jackson, M.; Mantsch, H. H. Beware of proteins in DMSO. Biochim. Biophys. Acta 1991, 1078, 231-235.

(31) Szymanska, A.; Hornowski, T.; Kozak, M.; Slosarek, G. The SAXS and rheological studies of HEWL amyloid formation. Acta Phys. Pol., A 2008, 114, 447-454.

(32) Castelletto, V.; Areas, E. P. G.; Areas, J. A. G.; Craievich, A. F. Effects of tetramethylurea on the tertiary structure of lysozyme in water. J. Chem. Phys. 1998, 109, 6133-6139.

(33) Carrotta, R.; Manno, M.; Giordano, F. M.; Longo, A.; Portale, G.; Martorana, V.; Biagio, P. L. S. Protein stability modulated by a conformational effector: effects of trifluoroethanol on bovine serum albumin. Phys. Chem. Chem. Phys. 2009, 11, 4007-4018.

(34) da Silva, M. A.; Areas, E. P. G. Solvent-induced lysozyme gels: Rheology, fractal analysis, and sol-gel kinetics. J. Colloid Interface Sci. 2005, 289, 394-401.

(35) Itakura, M.; Shimada, K.; Matsuyama, S.; Saito, T.; Kinugasa, S. A convenient method to determine the Rayleigh ratio with uniform polystyrene oligomers. J. Appl. Polym. Sci. 2006, 99, 1953-1959.

(36) Segre, P. N.; Pusey, P. N. Scaling of the dynamic scattering function of concentrated colloidal suspensions. Phys. Rev. Lett. 1996, 77, 771-774. 
(37) Liu, W.; Cellmer, T.; Keerl, D.; Prausnitz, J. M.; Blanch, H. W. Interactions of lysozyme in guanidinium chloride solutions from static and dynamic light-scattering measurements. Biotechnol. Bioeng. 2005, 90, 482490.

(38) Ma, R. J.; Wang, C. H. Studies of the protein protein-interaction of lysozyme in dimethylsulfoxide water solutions by quasielastic lightscattering. J. Phys. Chem. 1983, 87, 679-682.

(39) Liu, W.; Bratko, D.; Prausnitz, J. M.; Blanch, H. W. Effect of alcohols on aqueous lysozyme-lysozyme interactions from static lightscattering measurements. Biophys. Chem. 2004, 107, 289-298.

(40) Batas, B.; Jones, H. R.; Chaudhuri, J. B. Studies of the hydrodynamic volume changes that occur during refolding of lysozyme using sizeexclusion chromatography. J. Chromatogr., A 1997, 766, 109-119.

(41) Uversky, V. N. Use of fast protein size-exclusion liquid-chromatography to study the unfolding of proteins which denature through the molten globule. Biochemistry 1993, 32, 13288-13298.

(42) Kaatze, U.; Pottel, R.; Schafer, M. Dielectric Spectrum of DimethylSulfoxide Water Mixtures as a Function of Composition. J. Phys. Chem. 1989, 93, 5623-5627.

(43) Nicoli, D. F.; Benedek, G. B. Study of thermal-denaturation of lysozyme and other globular proteins by light-scattering spectroscopy. Biopolymers 1976, 15, 2421-2437.

(44) Glatter, O. New method for evaluation of small-angle scattering data. J. Appl. Crystallogr. 1977, 10, 415-421.

(45) Glatter, O. Interpretation of real-space information from small-angle scattering experiments. J. Appl. Crystallogr. 1979, 12, 166-175.

(46) Lindner, P.; Zemb, T. Neutron, X-rays and Light: Scattering Methods Applied to Soft Condensed Matter; Elsevier: Amsterdam, 2002.

(47) Svergun, D. I.; Richard, S.; Koch, M. H. J.; Sayers, Z.; Kuprin, S.; Zaccai, G. Protein hydration in solution: Experimental observation by x-ray and neutron scattering. Proc. Natl. Acad. Sci. USA 1998, 95, 2267-2272.

(48) Tardieu, A.; Le Verge, A.; Malfois, M.; Bonnete, F.; Finet, S.; RiesKautt, M.; Belloni, L. Proteins in solution: from X-ray scattering intensities to interaction potentials. J. Cryst. Growth 1999, 196, 193-203.

(49) Iwase, H.; Hirai, M.; Arai, S.; Mitsuya, S.; Shimizu, S.; Otomo, T.; Furusaka, M. Comparison of DMSO-induced denaturation of hen egg- white lysozyme and bovine alpha-lactalbumin. J. Phys. Chem. Solids 1999, 60, 1379-1381.

(50) Chen, L. L.; Hodgson, K. O.; Doniach, S. A lysozyme folding intermediate revealed by solution X-ray scattering. J. Mol. Biol. 1996, 261, 658-671.

(51) Doniach, S. Changes in biomolecular conformation seen by small angle X-ray scattering. Chem. Rev. 2001, 101, 1763-1778.

(52) Arai, S.; Hirai, M. Reversibility and hierarchy of thermal transition of hen egg-white lysozyme studied by small-angle X-ray scattering. Biophys. J. 1999, 76, 2192-2197.

(53) Kamatari, Y. O.; Konno, T.; Kataoka, M.; Akasaka, K. The methanol-induced transition and the expanded helical conformation in hen lysozyme. Protein Sci. 1998, 7, 681-688.

(54) Efimova, Y. M.; van Well, A. A.; Hanefeld, U.; Wierczinski, B.; Bouwman, W. G. Neutron and ion beams in biological research - On the scattering length density of proteins in H2O/D2O: Determination of H-D exchange using ES+ I-MS. J. Radioanal. Nucl. Chem. 2005, 264, 271275.

(55) Kamiyama, T.; Matsusita, T.; Kimura, T. Volumetric study of lysozyme in dimethyl sulfoxide plus water solution at $298.15 \mathrm{~K}$. J. Chem. Eng. Data 2003, 48, 1301-1305.

(56) Lipfert, J.; Doniach, S. Small-angle X-ray scattering from RNA, proteins, and protein complexes. Annu. Rev. Biophys. Biomol. Struct. 2007, $36,307-327$.

(57) McCarney, E. R.; Kohn, J. E.; Plaxco, K. W. Is there or isn't there? The case for (and against) residual structure in chemically denatured proteins. Crit. Rev. Biochem. Mol. Biol. 2005, 40, 181-189.

(58) Kohn, J. E.; Millett, I. S.; Jacob, J.; Zagrovic, B.; Dillon, T. M.; Cingel, N.; Dothager, R. S.; Seifert, S.; Thiyagarajan, P.; Sosnick, T. R.; Hasan, M. Z.; Pande, V. S.; Ruczinski, I.; Doniach, S.; Plaxco, K. W. Random-coil behavior and the dimensions of chemically unfolded proteins. Proc. Natl. Acad. Sci. USA 2004, 101, 12491-12496.

(59) Dill, K. A.; Shortle, D. Denatured states of proteins. Annu. Rev. Biochem. 1991, 60, 795-825.

(60) Fitzkee, N. C.; Rose, G. D. Reassessing random-coil statistics in unfolded proteins. Proc. Natl. Acad. Sci. USA 2004, 101, 12497-12502. 